A. Schardong, et al., Int. J. of Safety and Security Eng., Vol. 9, No. 1 (2019) 13-25

\title{
USE OF QUANTITATIVE RESILIENCE IN MANAGING URBAN INFRASTRUCTURE RESPONSE TO NATURAL HAZARDS
}

\author{
ANDRE SCHARDONG, SLOBODAN P. SIMONOVIC \& HOWARD TONG \\ Department of Civil and Environmental Engineering, The University of Western Ontario, London, Canada.
}

\begin{abstract}
Damages to urban systems as a result of various natural hazards have escalated in recent years. The observed trend is expected to increase in the future as the impacts of population growth, rapid urbanization and climate change persist. To alleviate the damages associated with these impacts, it is recommended to integrate disaster management methods into planning, design and operational policies under all levels of government. This manuscript proposes the use of quantitative resilience concept (dynamic in time and space) to assess the response of an urban system to natural hazards. The implementation of the concept has been done in the form of the web-based decision support system that operates in near real-time. It is designed to assist decision makers in selecting the best options for integrating adaptive capacity into their communities to protect against the negative impacts of hazards. The tool is developed for application in Toronto, Ontario, Canada.

Keywords: adaptation, decision support, disaster management, hydro-meteorological, online tool, Resilience, urban systems
\end{abstract}

\section{INTRODUCTION}

Natural hazards have caused an increasing amount of damage to urban systems in the recent years. The observed trend is expected to increase in the future as the impacts of population growth, rapid urbanization, and climate change persist. Natural disasters have become more frequent and damaging to physical and socioeconomic environments in recent years and decades [1]. In the last decade approximately $\$ 4$ trillion of direct economic damages have been attributed to natural disasters globally. This does not account for the loss of life and cultural assets that are difficult to evaluate [2,3]. Furthermore, in last two decades the estimated annual economic losses caused by natural disasters have increased from $\$ 50$ billion to $\$ 180$ billion. Most of these losses are associated with extreme weather events such as floods, droughts, landslides, cyclones, extreme temperature and others [2]. According to [4], in 2015 alone, 346 significant disasters were linked to natural hazards, and an estimated 22,500 fatalities and over $\$ 66$ billion economic damages were lost.

The rising number of natural disasters that have occurred in recent years may be linked to three key factors including climate change, rapid and sometimes poorly planned land use change and population growth, especially mass urbanization. Climate change is believed to increase the frequency and intensity of extreme precipitation events. The average global temperature is projected to rise significantly, disrupting the balance of the climate system and hydrological cycle, causing unprecedented extreme meteorological events that will overwhelm critical infrastructure capacity as current design [1].

Integrating disaster management methods into planning, design and operational policies may aid or mitigate the damages induced by hazard events. In this manuscript, we propose the use of the quantitative resilience concept to assess the response of an urban system to hazardous event and how this changes as a result of varying adaptation measures.

Traditional risk management is defined as the combination of three elements: (1) probability of an extreme event; (2) exposure of infrastructure or human elements; and (3) susceptibility 
of people, property, infrastructure, to damage caused by an extreme event [1,2]. There has been a shift from the traditional risk-based approach to resilience-based disaster management $[5,6]$.

Decision support systems (DSS) are used to provide meaningful inputs for improved decision making in complex domains. The DSS tool created by [1] named ResilSIM, to the best knowledge of the authors, was one of the first tools to use resilience as a measure of the urban system's ability to function in the event of a hazard as well as recover from the damages.

The objective of this manuscript is to present the evolution of the ResilSIM tool and introduce the updated version, ResilSIM v2. The implementation has been done in the form of the web-based and mobile friendly DSS. It is designed to assist decision makers in experimenting with different adaptation in order to mitigate the negative impacts of a hazard. The tool is developed for general application with user input data capabilities combined with the open source OpenStreetMap GIS database. One potential application for the Toronto, Ontario, Canada is presented.

The remainder of the manuscript is organized as follows: the method including the resilience definition, research objectives and methodological background are presented in section 2; a case study of the ResilSIM v2 tool in Toronto, Ontario is demonstrated in section 3; and finally, recommendations for future work are provided in section 4 .

\section{METHODOLOGY}

In this section: (i) the resilience measure is defined; (ii) the objectives of ResilSIM v2 are identified; (iii) the methodological background is outlined; and (iv) the decision support tool features are described.

\subsection{Resilience Definition}

The IPCC defines resilience, in the context of disaster management, as "the ability of a system and its component parts to anticipate, absorb, accommodate or recover from the effects of a hazardous event in a timely and efficient manner, including through ensuring the preservation, restoration or improvement of its essential basic structures and functions" [3]. The definition of risk is the susceptibility of the system and its components to damage caused by hazards scenarios. Traditional risk is a static metric that does not change overtime. Resilience is a dynamic metric that is capable of presenting system performance over the duration of a disaster event.

According to [5], [7], [8], and [9] a resilient system may be achieved by choosing a sufficient set of adaptation measures. Recently, [5], introduced the dynamic resilience metric using simulation model integrated with GIS processing, similar to the procedure adopted by [1]. In [10], a comparison of static and dynamic resilience applied to multipurpose reservoir operation is presented. Their results show that the dynamic resilience is a powerful tool for selecting proactive and reactive adaptive response to hazard events that could not be achieved using the traditional risk based approach. The dynamic resilience concept is expanded by [11] using network theory and applied to the city of Toronto infrastructure as a case study. These concepts are incorporated into the current version of the ResilSIM v2 for the quantification of the dynamic resilience metric.

\subsection{Objectives of ResilSIM v2 tool}

The ResilSIM v2 is a web-based tool that allows (i) user input for representing physical and socioeconomic urban systems and (ii) assessment of its resilience to natural or human made 
disasters. It can assist decision makers in selecting appropriate adaptation options that will maximize urban system resilience to a hazardous event.

The tool can function with a variety of datasets that describe key urban infrastructures, critical facilities (such as hospitals, schools, ambulance, fire and police stations), land use characteristics and social infrastructure. It is paramount that geographic information is associated with the data. Network data format are allowed, and specialized algorithms are employed to calculate the resilience metric. Infrastructure, social data, buildings, is mostly publicly available. However, private data can be incorporated into the tool database by the user for the resilience calculation as well.

The ResiSIM v2 can simulate one or more hazard scenarios, defined by the users through the user interface and GIS tool to project the hazard event for the region and compute an initial value of the urban system's resilience to the disturbance. The tool offers the user the possibility of creating a list of proactive and reactive measures of adaptive capacity that will be applied to improve the system performance and resilience. The user can select an adaptation option to be implemented virtually and observe how community resilience is affected. After the adaptation options are defined, the resilience is re-computed and compared to its initial value, serving as a basis for comparison for potential upgrades to the system.

The tool enables users to quickly make decisions that can reduce the physical, social and economic consequences associated with the hazards. These include damages to the built and natural environments (roads, housing, buildings, urban systems) as well as the danger and disruption posed to human welfare.

\subsection{Methodological Background}

The ResilSIM v2 is build as a generalized system allowing the calculation of Resilience metrics using a variety of datasets that describe key urban infrastructures, critical facilities, land use characteristics and social infrastructure. The tool mainly utilizes two types of data inputs: i) network data format, with a formal structure containing nodes connected by links or edges, and for which, specialized algorithms are employed to calculate the resilience metric; ii) other GIS data layers containing information about urban infrastructure, social data, buildings, critical facilities and others. For the network layers, each element component may have or its own physical capacity (usually represented as cost on a link for the mathematical representation).

The physical capacity measurement consists of the infrastructure's structural integrity or a unit related to its functionality. This is also the step related to the input of dependencies between network components. Dependencies can be classified as intra-dependent, a node or edge within a network dependent on another node or edge within the same network type, or interdependent, a node or edge within a network dependent on another node or edge from a different network type. GIS data layers have flexibility to incorporate other data types, such as point, line and polygon shapes.

The ResilSIM v2 dataflow and case analysis can be summarized in a few steps, as follows:

1. Description of Infrastructure System: on step the data is provided, i.e., network data, infrastructure data in form of GIS layers, social information and any other information or data the user judges relevant;

2. Hazards Input: definition of the hazard scenarios and input of the affected areas and regions and the magnitude on time and space; 
3. Impact Assessment: the impact on the urban infrastructure (networks and data layers) caused by the hazards.

4. Adaptation Options: adaptation options are defined for each layer and hazards and will impact (improve) the resilience metric;

5. Assessment of System Performance and Resilience: Assessment of the Resilience and the impacts of the adaptation options.

Hazards are characterized by their damage area and magnitudes. They are inputs from the user. The tool allows the user to change the hazard affected area and severity over time to demonstrate the progression of the hazard. The impact assessment step then uses damage functions to estimate the reduction in capacity for the various system elements.

User input adaptation options can then be specified to demonstrate how system component behaviors shift if they are implemented. These can be classified as pre-disaster measures that change the disaster impact relationship for the component or post disaster measures that speed up system element recovery. The ResilSIM v2 tool integrates physical, social and economic impacts of disaster events and adaptation options implemented to an urban system into a single measure known as resilience, $R$. The procedure for computing the resilience metric is adapted from [1], [6], [11] and [12].

The overall system resilience calculation for each time step is illustrated on the flowchart presented in Fig. 1. The physical performance of individual networks and layers is aggregated to form a single network/layer system performance and resilience. Once this process is repeated for all infrastructure, it is aggregated further to calculate the overall system resilience.

An example of typical system performance curve (black line) is presented in Fig. 2. The shaded area under the curve represents the loss of system performance caused by the hazard over time. The $\mathrm{x}$-axis represents time, where $t_{0}$ and $t_{l}$ represent the time at the beginning and end of the disaster event respectively. The system performance drops once the disaster begins at $t_{0}$ and recovers over time.

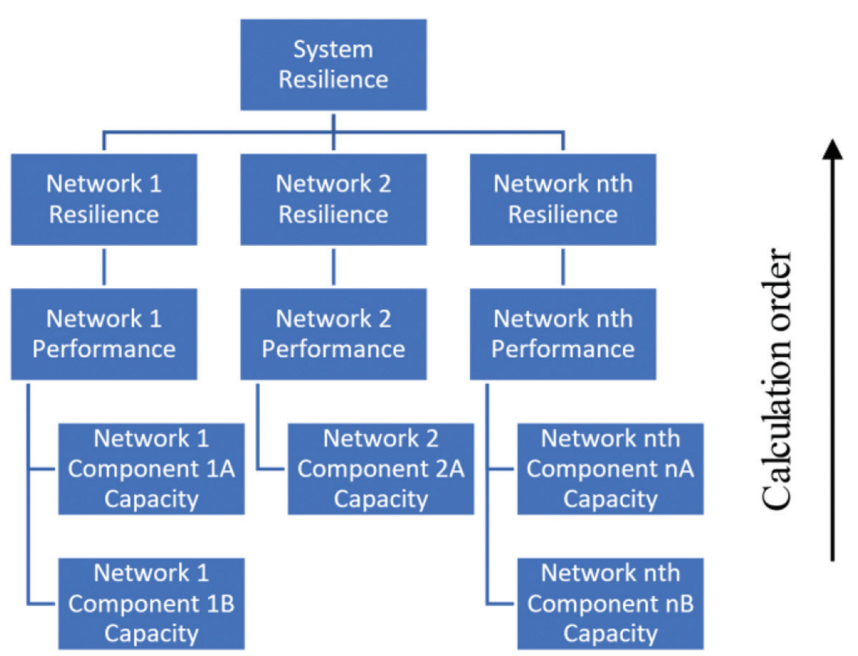

Figure 1: Resilience calculation order and flowchart of the ResilSIM v2 
The area above the system performance curve represents the loss in system performance over time $\left(\rho^{i}\right)$. The area spans from the initial time of disturbance to the time at the end of system recovery and is calculated as:

$$
\rho^{i}(t)=\int_{t_{0}}^{t}\left[P_{0}^{i}-P^{i}(t, s)\right] d t \text { where } t \in\left[t_{0}, t_{r}\right]
$$

where $P_{0}^{i}$ is the is the initial system performance level, $i$ the hazard, $P^{i}(t, s)$ represents measure of system performance for each time step $t$ and location in space $s$, and $t r$ the end of the recovery process.

The resilience of each system component, $r^{i}(t, s)$, represented by the area below the system performance curve in Fig. 2 is calculated using:

$$
r^{i}(t)=1-\left(\frac{\rho^{i}(t)}{P_{0}^{i} \times\left(t-t_{0}\right)}\right)
$$

The resilience of each system component can be combined into the final, system resilience using:

$$
\mathrm{R}(t, s)=\left\{\prod_{i=1}^{M} r^{i}(t, s)\right\}^{\frac{1}{M}}
$$

where $M$ is the number of hazards.

Figure 3 illustrates one representation of the four components of resilience graphically. The resilience components include robustness, redundancy, resourcefulness and rapidity. Based on the mathematical representations of the four resilience components from [10], the tool can estimate a value for them with the resilience curve(s) generated.

The application of the methodology is demonstrated using OpenStreetMap (OSM) data and other publicly available data for the city of Toronto. Two theoretical hazard scenarios are created to showcase the use of the tool and the calculation of the system performance and resilience.

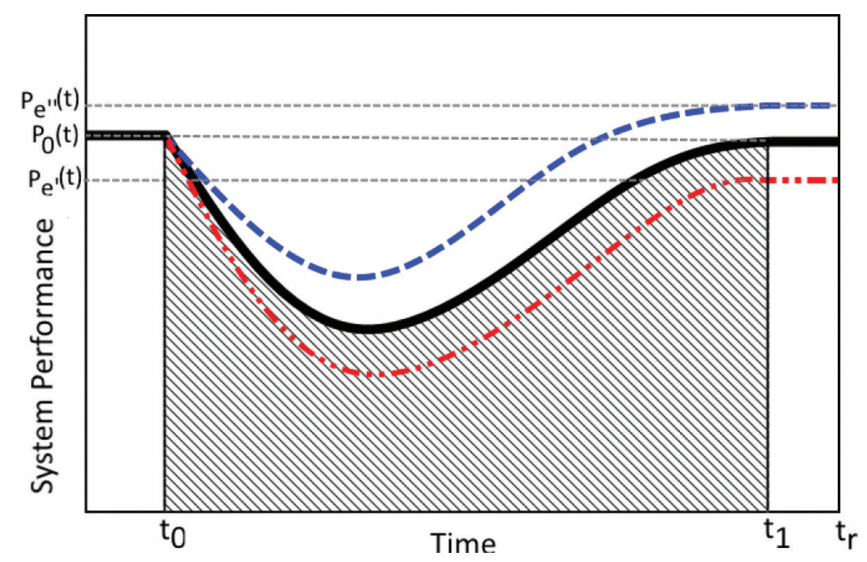

Figure 2: System performance to a disturbance (partial recovery, recovery to pre-hazard system performance, and strengthening of system performance) (adapted from [10]) 


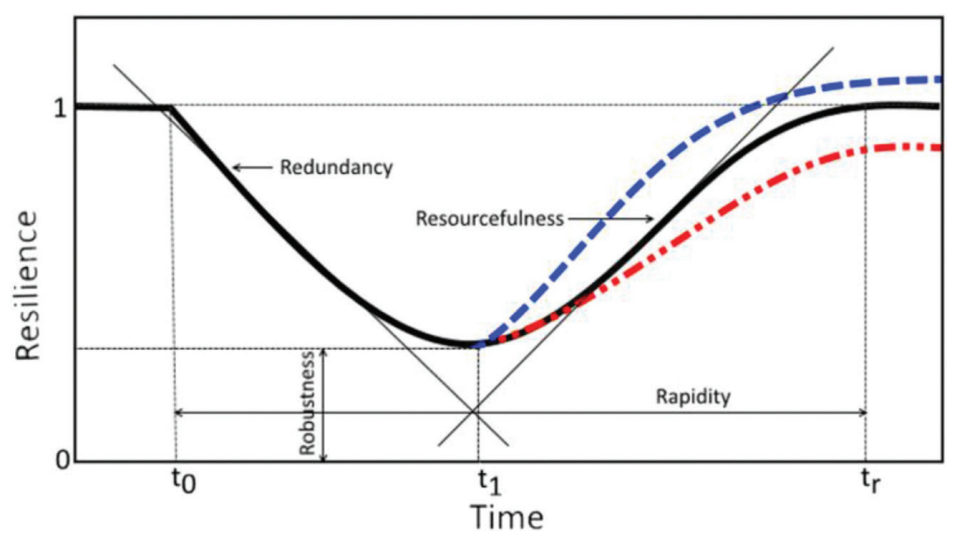

Figure 3: System performance to a disturbance (partial recovery, recovery to pre-hazard system performance, and strengthening of system performance) (adapted from [10])

\subsection{Decision Support System Description}

The ResilSIM v2 tool is implemented as a generalized decision support system (DSS) that calculates the system performance and resilience to one or more extreme events that disrupt urban systems. The following section describes the major components of the tool: (i) user interface; (ii) database and (iii) mathematical module.

The procedure for calculating the resilience within the ResilSIM v2 tool can be described in following steps: (1) selection of the analysis (city, or urban center); (2) organization of the data for input into the tool: layer and networks representing urban system such as buildings, critical facilities, energy, and others, as well as social data; (3) input of the hazard scenarios and damage functions; (4) definition and input of the adaptation measures; (5) simulation of the hazardous event and the computation of the resilience metric.

\subsubsection{User Interface}

The user interface, as presented in Fig. 4, has the following components: (i) GIS mappping component used to present geographic data and assist user's input; (ii) data input functionalities that allow users to select a set of adaptation measures; and (iii) results visualization - functionalities for the presentation of the system performance as interactive graphs, and tables to export the data extracted from the database.

The GIS interface is used in the resilience calculation screen to aid the user selecting a region of the city for the resilience calculation. Additionally, it is used to present all the geographic layers used in the tool's database. The adaptation measures controls are built as simple sliders where the user can select from a range between a minimum and maximum value defined for each measure.

\section{Data: OSM, Layers, Networks}

The tool has several pre-loaded layers that are imported from the OpenStreetMap (OSM) dataset [13]. The OSM is open-sourced dataset and is available with high level of details, especially for larger urban centers. The user can use this set to start the analysis without providing additional data. Some of the layers included are: roads, buildings, land use, critical facilities and railways. Other layers with geographical information can be provided, as well as social data and network-based layers. 


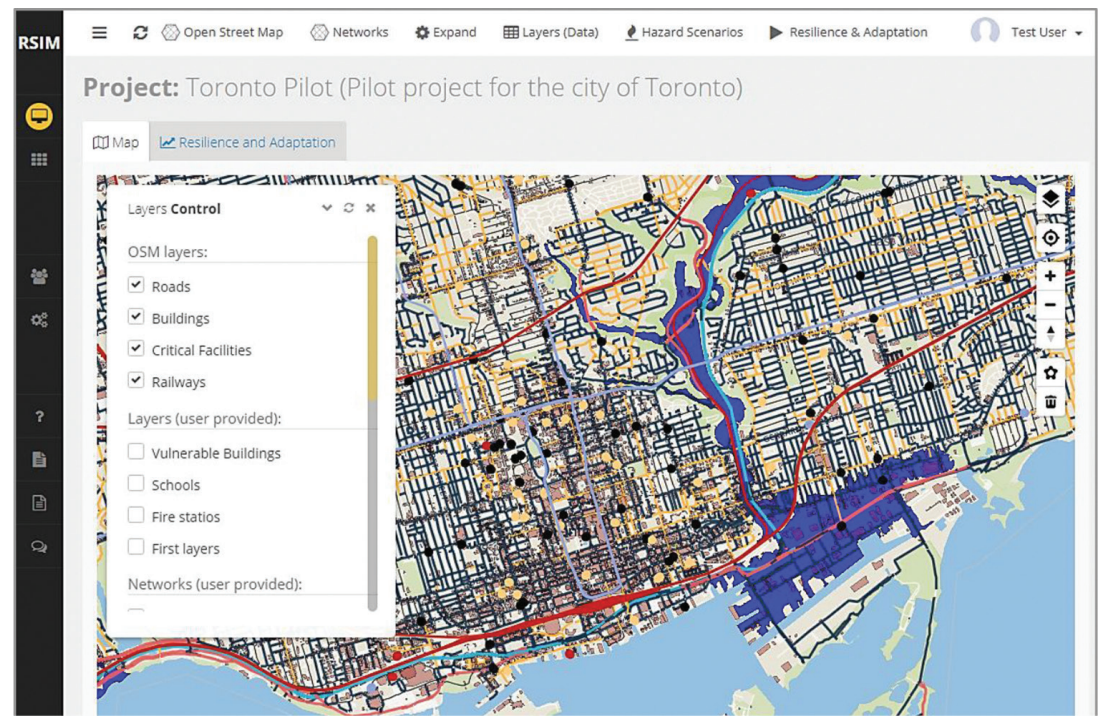

Figure 4: The ResilSIM v2 tool user interface

\section{Hazard Scenarios}

Several different types of disaster scenarios are supported by the ResilSIM v2: flooding, hurricanes (strong winds), fire and extreme temperature. The hazard scenarios are defined by de user using the GIS interface and both location or extent of the disaster and its duration have to be provided, as well as the magnitude of the event for each time step.

\section{Adaptation Measures}

Following similar concepts as the previous version, ResilSIM v2 classifies the options in two categories: (i) Reactive adaptation measures that are implemented during the disaster event; and (ii) Proactive adaptation measures that are implemented in advance of the disaster event.

Proactive adaptation measures intend to reduce the loss in system performance (over the duration of the disturbance) and improve resilience. On the other hand, reactive adaptation options, improve the rapidity of the recovery of system performance to a normal level of function, also enhancing the value of resilience. Evidently, the urban system performance returns to a normal level of function faster after the real-time adaptation options have been introduced.

\subsubsection{Database}

The database system stores the OpenStreetMap dataset, DEM data and several other geographic data, such as land use layers, engineering data, provided by the users and the tool's configuration parameters. The database management system (DBMS) used is the latest version of PostgreSQL with the PostGIS extension.

\subsubsection{Mathematical module}

The ResilSIM v2 incorporates a mathematical module that provides support for computation of system performance and resilience metrics, based on the methodology presented. This module also incorporates the codes and logic to extract and process the data from the database. 


\section{CASE STUDY}

The tool is capable of estimating community resilience for a variety of natural or human disasters. The tool is demonstrated for application in the city of Toronto, Ontario that is vulnerable to climate change caused flooding. This section provides a brief description of the data used, the hazard scenarios and damage functions created and demonstration of the use of adaptation options; and (iii) demonstration of model output for this specific case study.

\subsection{Hazard Scenario and Impact assessment}

The ResilSIM v2 tool allow the input of supplementary data (to the ready to use OpenStreetMap layers) that represent urban systems in the GIS or network format. Similarly, several hazards can be defined and will be used for the calculation of the resilience. The hazards are created by providing the duration, the affected areas (using the GIS API) and the magnitudes of the hazard over the duration of the event (Fig. 5).

The ResilSIM v2 tool is also flexible regarding the input and impact assessment for each of the hazards created. The impacts can be provided as: (1) damage tables; (2) damage equations and (3) fragility curves (Figs 5 and 6).

\subsection{Data and parameters of the simulation}

For the simulation presented, a few layers from the Open Street map dataset were selected: roads, buildings and critical facilities (schools, police stations and fire stations). Additional geographical enabled data, provided by the City of Toronto and Toronto Hydro was used: digital elevation model for the city of Toronto, power supply network provided by Toronto Hydro (transformers and supplied areas), as well as additional critical facilities (schools, hospitals, fire stations and police stations). Two hazards were defined as one flooding and windstorm event, affecting the downtown area of the city of Toronto, Canada as presented in Fig. 9 (red polygons), and the hypothetical test case hazards were defined with a duration of approximately 24 hours.

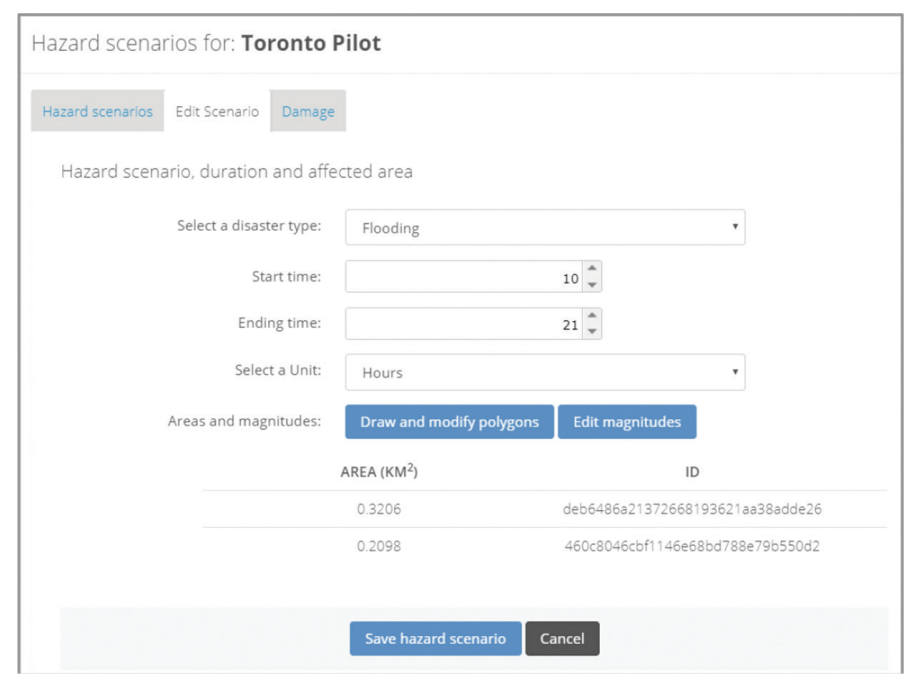

Figure 5: Characteristics of a hazard scenario in the ResilSIM v2 tool. 


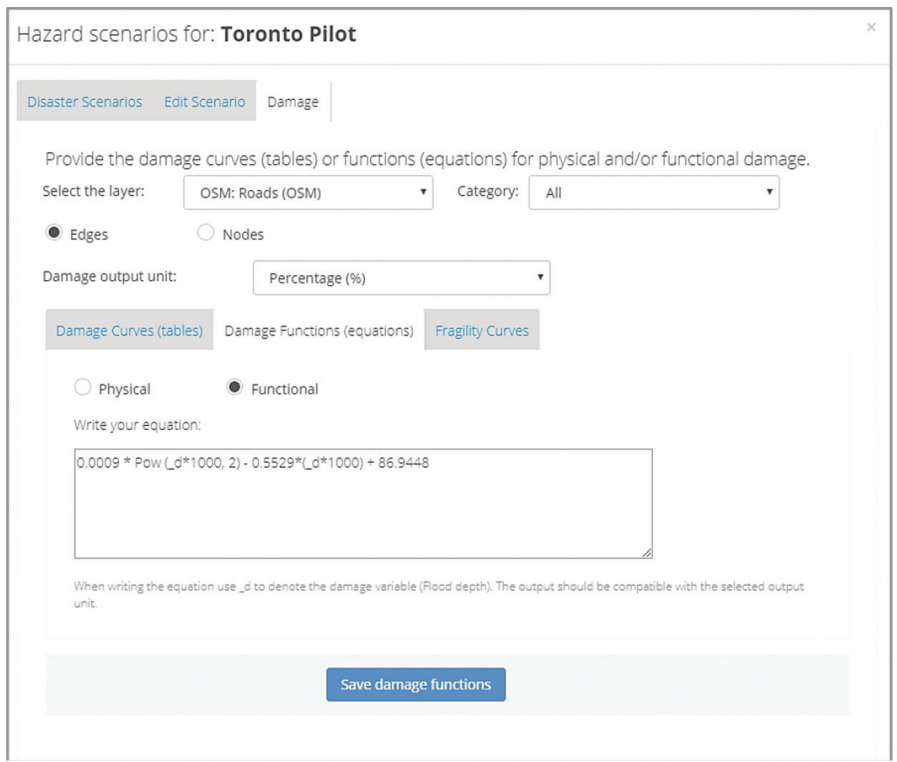

Figure 6: Damage input for the various hazards scenarios created by the user.

\subsection{Adaptation Options}

Increase in adaptive capacity of an urban system reduces the loss of system performance over the duration of the hazard and the recovery time, and as a result, the system resilience is improved. The ResilSIM v2 allows adaptation options to be freely defined (Figs 7 and 8) for each of the data layers and networks pre-loaded in the database or provided by the user. The adaptation options will improve the overall performance of an urban system when subject to hazards and the user will be able to evaluate their efficiency.

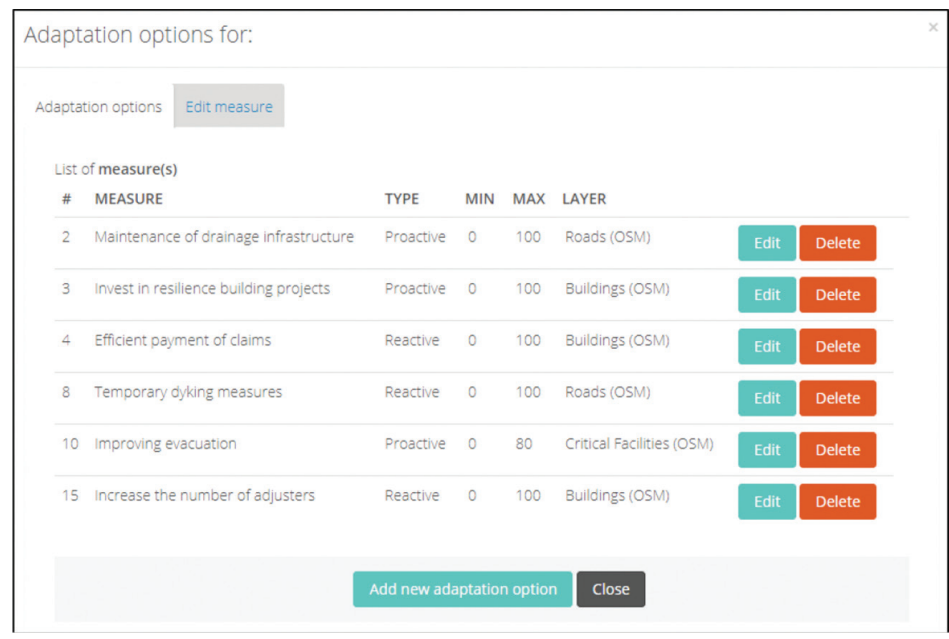

Figure 7: Adaptation options defined by the user in the ResilSIM v2 user interface. 


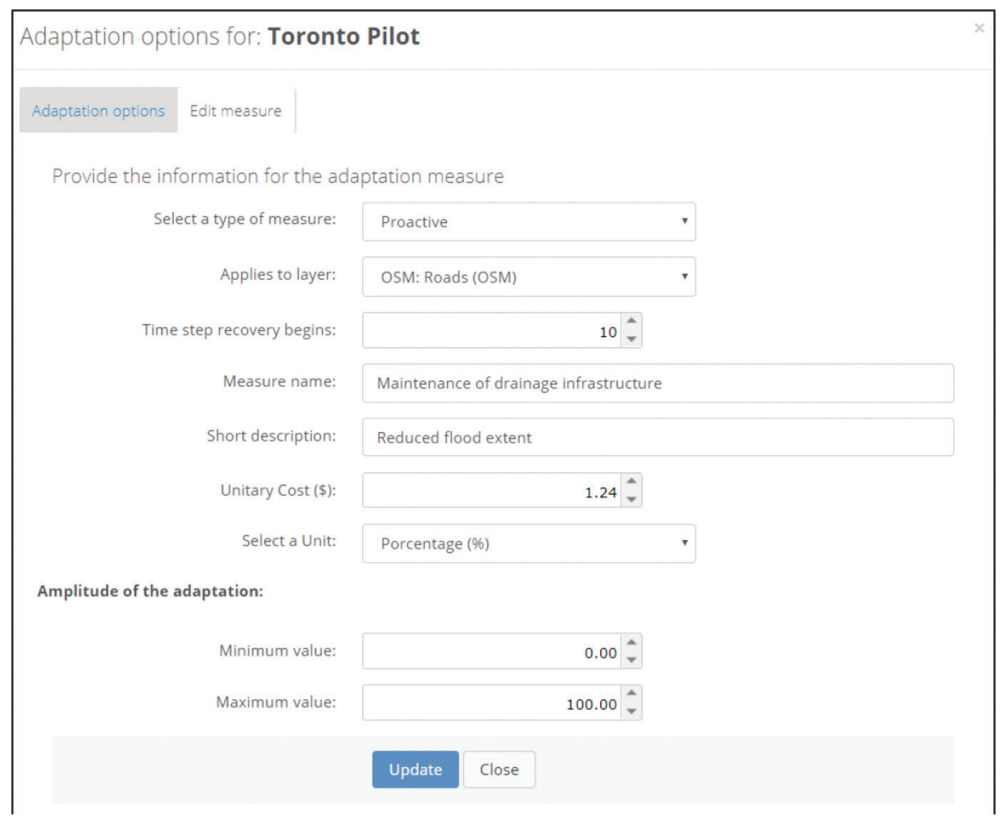

Figure 8: Characteristics of the adaptation options in the ResilSIM v2.

\subsection{System Performance and Resilience simulation}

The user's inputs for the hazard scenarios limit the geographic extension of the resilience calculation in the tool. Figure 9 shows the combined disaster regions (light red shaded areas) from different scenarios for a hypothetical case study. These regions will then be used by the tool's mathematical models to calculate the loss of system performance over time and ultimately the combined system resilience.

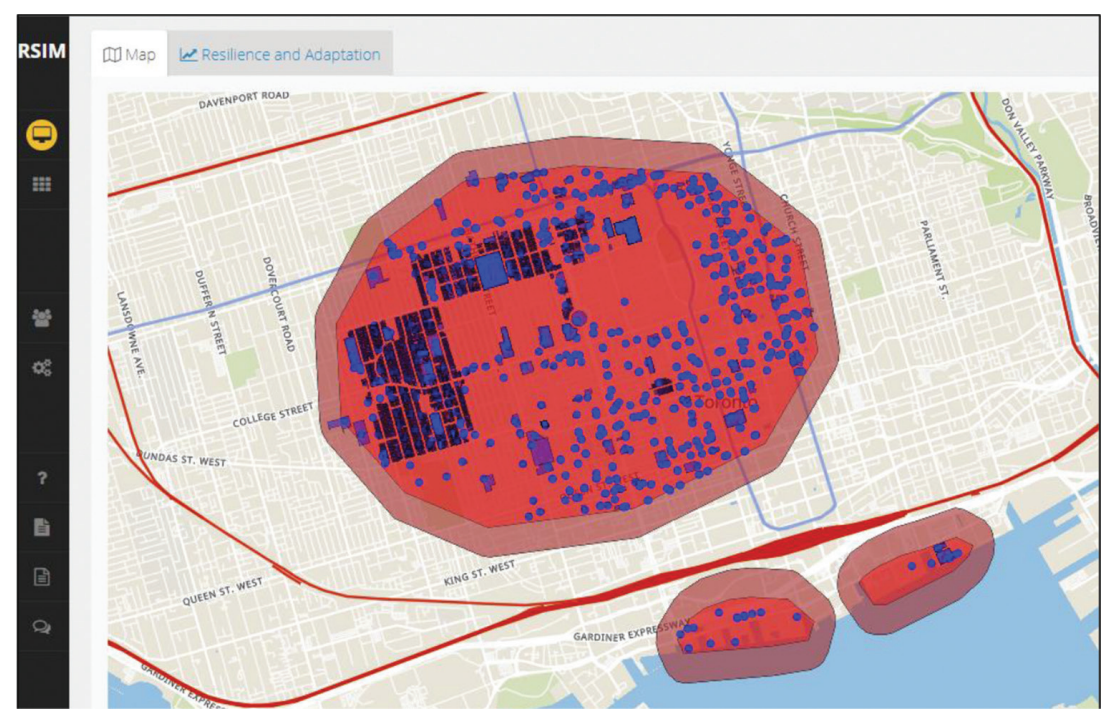

Figure 9: Geographic extension of the area defined by the hazards from the user's inputs. 
After defining the hazard scenarios and damage functions, the tool calculates the system performance measures and the combined resilience metric using the methodology presented (Fig. 10).

The pro-active or reactive adaptation measures defined by the user for each layer and disaster scenario will impact the resilience calculated by the tool. The selection of the adaptation measures is controlled by a set of sliders with upper and lower limits, as defined in the input scenarios (right hand side of Figs 10 and 11). The user can quickly evaluate the impact of changing the values of the adaptation options on the system's resilience and, based on the response, identify measure/s with high efficiency in improving resilience in response to the hazard scenario.

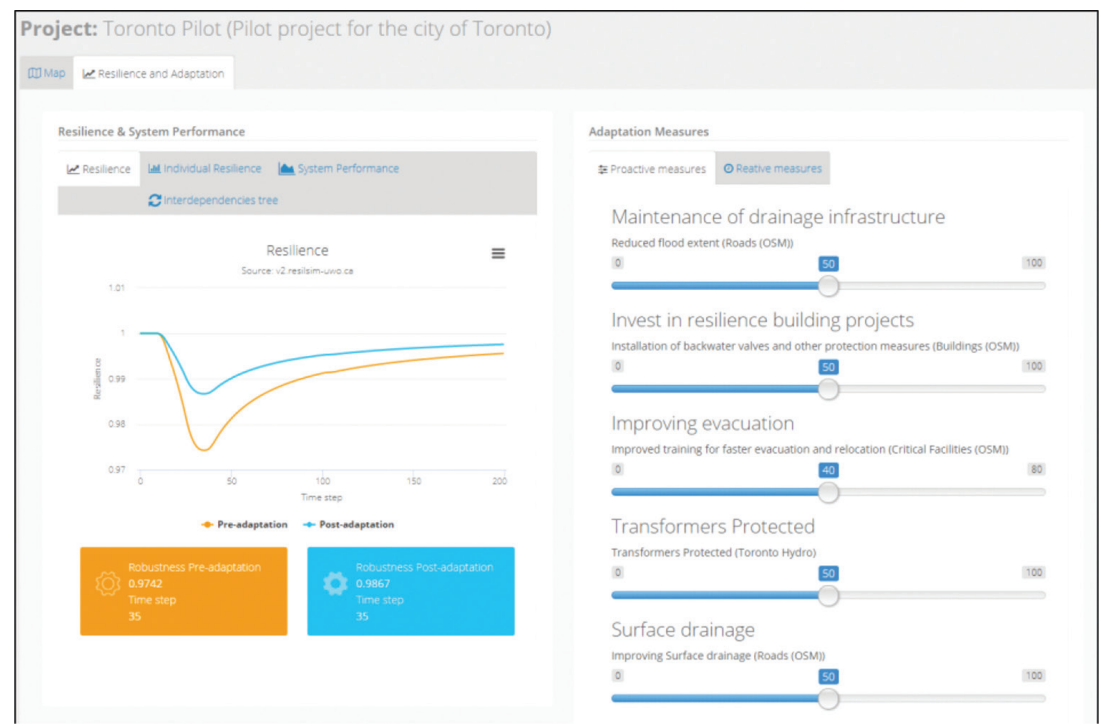

Figure 10: Pro-active adaptation measures applied to the system and response of the resilience. The orange line shows the system resilience without adaptation and the blue line with pro-active and reactive adaptation measures.

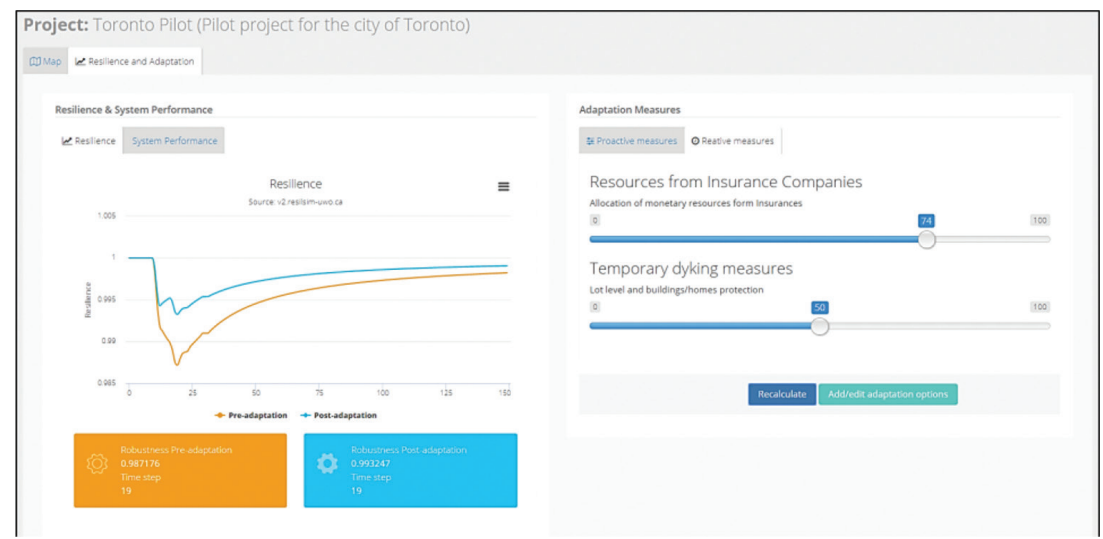

Figure 11: Reactive adaptation measures applied to the system and response of the Resilience metric. The orange line shows the system resilience without adaptation and the blue line with pro-active and reactive adaptation measures. 


\section{CONCLUSION}

This manuscript presents a decision support tool, ResilSIM v2, that is able to estimate the resilience of an urban system exposed to the impacts of various types of hazards, such as floods, hurricanes, fire, and others. The tool uses an open source GIS dataset as starting point for the analysis. The user, however, has the ability to incorporate additional data in form of GIS layers and networks to represent the physical and socioeconomic components of the urban system. Using spatial programming techniques, the resilience metric is computed as an amalgamation of system performance measures with respect to various impacts of the hazard scenarios.

The ResilSIM v2 calculates an initial value of resilience as basis for comparison. The user can subsequently add and experiment with different proactive and reactive adaptation measures and compare their impact on the system resilience.

The tool is tested with data for the city of Toronto, Canada, however, its generalized nature allows it to be applied to any urban center. Hazard scenarios and adaptation measures are user defined inputs allowing a various combinations of disaster and recovery strategies to be evaluated.

Resilience is an innovative disaster management measure that can assist decision makers in selecting and prioritizing actions for upgrading urban systems with high exposure to extreme events.

\section{ACKNOWLEDGEMENTS}

The authors would like to acknowledge the financial support for the presented research provided by Chaucer Syndicates (London, UK) and Natural Sciences and Engineering Research Council of Canada (NSERC) to the second author.

\section{REFERENCES}

[1] Irwin, S., Schardong, A., Simonovic, S.P. \& Nirupama, N., ResilSIM - A decision support tool for estimating resilience of urban systems. Water, 8, p. 377, 2016. https://doi.org/10.3390/w8090377

[2] World Bank. Disaster Risk Management: Overview, 2015, available at http://www. worldbank.org/

[3] IPCC. Summary for Policymakers. Managing the Risks of Extreme Events and Disasters to Advance Climate Change Adaptation, ed. C.B. Field, V. Barros, T.F. Stocker, D. Qin, D.J. Dokken, K.L. Ebi, M.D. Mastrandrea, K.J. Mach \& G.K. Plattner, Cambridge University Press, Cambridge, 2012.

[4] World Health Organization. Health Emergency and Disaster Risk Management: Overview, available at http://www.worldbank.org/, 2017.

[5] Simonovic, S.P., From risk management to quantitative disaster resilience: a paradigm shift. International Journal of Safety and Security Engineering, 6, pp. 85-95, 2016. https://doi.org/10.2495/safe-v6-n2-85-95

[6] Simonovic, S.P. \& Peck, A., Dynamic resilience to climate change caused natural disasters in coastal megacities quantification framework. British Journal of Environment and Climate Change, 3, pp. 378-401, 2013. https://doi.org/10.9734/bjecc/2013/2504 
[7] Bruneau, M., Chang, S.E., Eguchi, R.T., Lee, G.C., O'Rourke, T.D., Reinhorn, A.M., Shinozuka, M., Tierney, K., Wallace, W.A. \& von Winterfeldt, D., A framework to quantitatively assess and enhance the seismic resilience of communities. Earthquake Spectra, 19(4), pp. 733-752, 2003. https://doi.org/10.1193/1.1623497

[8] Chang, S.E. \& Shinozuka, M., Measuring improvements in disaster resilience of communities. Earthquake Spectra, 20(3), pp. 739-755, 2004. https://doi.org/10.1193/1.1775796

[9] Cutter, S.L., Barnes, L., Berry, M., Burton, C., Evans, E., Tate, E. \& Webb, J., A place-based model for understanding community resilience to natural disasters. Global Environmental Change, 18, pp. 598-606, 2008. https://doi.org/10.1016/j.gloenvcha.2008.07.013

[10] Simonovic, S.P. \& Arunkumar, R., Comparison of static and dynamic resilience for a multipurpose reservoir operation. Water Resources Research, 52, pp. 8630-8649, 2016. https://doi.org/10.1002/2016WR019551

[11] Kong, J. \& Simonovic, S.P., Multi-Hazard Resilience Model of an Interdependent Infrastructure System (Water Resources Research Report no. 102), Facility for Intelligent Decision Support, Department of Civil and Environmental Engineering, London, Ontario, Canada, 2017.

[12] Peck, A. \& Simonovic, S.P., Coastal Cities at Risk (CCaR): Generic System Dynamics Simulation Models for Use with City Resilience Simulator Final Report (Water Resources Research Report No. 083) Facility for Intelligent Decision Support, Department of Civil and Environmental Engineering: London, ON, Canada, 2013.

[13] OSM. The OpenStreetMap Project, available at http://openstreetmap.org, 2018. 